\title{
Idiopathic steroid-sensitive nephrotic syndrome
}

INSERM

\section{Source}

INSERM. (1999). Orphanet: an online rare disease and orphan drug data base. Idiopathic steroid-sensitive nephrotic syndrome. ORPHA:69061

Steroid-sensitive nephrotic syndrome (SSNS) is a kidney disease defined by selective proteinuria, hypoalbuminaemia and, on renal biopsy, minimal changes without immunoglobulin deposits. 